\title{
Health Literacy and Hospital Length of Stay: An Inpatient Cohort Study
}

\author{
Ethan G. Jaffee, MD¹, Vineet M. Arora, MD, MAPP², Madeleine I. Matthiesen, MD $^{3}$, \\ David O. Meltzer, MD, $\mathrm{PhD}^{4}$, Valerie G. Press, $\mathrm{MD}, \mathrm{MPH}^{2 \star}$
}

\begin{abstract}
${ }^{1}$ Psychiatry, Massachusetts General Hospital/McLean Hospital, Boston, Massachusetts; '2Section of General Internal Medicine, Department of Medicine, University of Chicago, Chicago, Illinois; ${ }^{3}$ Medicine-Pediatrics, Massachusetts General Hospital, Boston, Massachusetts; ${ }^{4}$ Section of Hospital Medicine, Department of Medicine, University of Chicago, Chicago, Illinois.
\end{abstract}

BACKGROUND: Associations between low health literacy (HL) and adverse health outcomes have been well documented in the outpatient setting; however, few studies have examined associations between low $\mathrm{HL}$ and in-hospital outcomes.

OBJECTIVE: To compare hospital length of stay (LOS) among patients with low $\mathrm{HL}$ and those with adequate $\mathrm{HL}$.

DESIGN: Hospital-based cohort study.

SETTING: Academic urban tertiary-care hospital.

PATIENTS: Hospitalized general medicine patients.

MEASUREMENTS: We measured HL using the Brief Health Literacy Screen. Severity of illness and LOS were obtained from administrative data. Multivariable linear regression controlling for illness severity and sociodemographic variables was employed to measure the association between HL and LOS.
RESULTS: Among 5540 participants, 20\% (1104/5540) had low HL. Participants with low HL had a longer average LOS (6.0 vs 5.4 days, $P<0.001)$. Low $\mathrm{HL}$ was associated with an $11.1 \%$ longer LOS (95\% confidence interval [Cl], 6.1\%-16.1\%; $P<0.001)$ in multivariate analysis. This effect was significantly modified by gender $(P=0.02)$. Low $\mathrm{HL}$ was associated with a $17.8 \%$ longer LOS among men $(95 \% \mathrm{Cl}, 10.0 \%-25.7 \%$; $P<0.001)$, but only a $7.7 \%$ longer LOS among women $(95 \%$ $\mathrm{Cl}, 1.9 \%-13.5 \% ; P=0.009)$.

CONCLUSIONS: In this single-center cohort study, low HL was associated with a longer hospital LOS. The findings suggest that the adverse effects of low HL may extend into the inpatient setting, indicating that targeted interventions may be needed for patients with low HL. Further work is needed to explore these negative consequences and potential mitigating factors. Journal of Hospital Medicine 2017;12:969-973. Published online first September 20, 2017. (C) 2017 Society of Hospital Medicine
Health literacy (HL), defined as patients' ability to understand health information and make health decisions, ${ }^{1}$ is a prevalent problem in the outpatient and inpatient settings. ${ }^{2,3}$ In both settings, low HL has adverse implications for self-care including interpreting health labels ${ }^{4}$ and taking medications correctly. ${ }^{5}$ Among outpatient cohorts, HL has been associated with worse outcomes and acute care utilization. ${ }^{6}$ Associations with low HL include increased hospitalizations, ${ }^{7}$ rehospitalizations, ${ }^{8,9}$ emergency department visits, ${ }^{10}$ and decreased preventative care use. ${ }^{11}$ Among the elderly, low HL is associated with increased mortality ${ }^{12}$ and decreased self-perception of health. ${ }^{13}$

A systematic review revealed that most high-quality $\mathrm{HL}$ outcome studies were conducted in the outpatient setting. ${ }^{6}$ There have been very few studies assessing effects of low HL in an acute-care setting. ${ }^{7,14}$ These studies have evaluated postdischarge outcomes, including admissions or readmissions, ${ }^{7-9}$ and medication knowledge. ${ }^{14}$ To the best of our knowledge, there are no studies evaluating associations

\footnotetext{
*Address for correspondence and reprint requests: Valerie G. Press, MD, MPH, 5841 South Maryland Avenue, MC 2007, Chicago, IL 60637; Telephone: 773-702-5170; Fax: 773-795-7398; E-mail: vpress@medicine.bsd.uchicago.edu

Received: March 8, 2017; Revised: May 10, 2017;

Accepted: May 19, 2017
}

2017 Society of Hospital Medicine DOI 10.12788/jhm.2848 between $\mathrm{HL}$ and hospital length of stay (LOS).

LOS has received much attention as providers and payers focus more on resource utilization and eliminating adverse effects of prolonged hospitalization. ${ }^{15}$ LOS is multifactorial, depending on clinical characteristics like disease severity, as well as on sociocultural, demographic, and geographic factors. ${ }^{16}$ Despite evidence that LOS reductions translate into improved resource allocation and potentially fewer complications, there remains a tension between the appropriate LOS and one that is too short for a given condition. ${ }^{17}$

Because low HL is associated with inefficient resource utilization, we hypothesized that low HL would be associated with increased LOS after controlling for illness severity. Our objectives were to evaluate the association between low $\mathrm{HL}$ and LOS and whether such an association was modified by illness severity and sociodemographics.

\section{METHODS}

\section{Study Design, Setting, Participants}

An in-hospital, cohort study design of patients who were admitted or transferred to the general medicine service at the University of Chicago between October 2012 and November 2015 and screened for inclusion as part of a large, ongoing study of inpatient care quality was conducted. ${ }^{18}$ Exclusion criteria included observation status, age under 18 years, non-English speaking, and repeat participants. Those who died during hospitalization or whose discharge status 
was missing were excluded because the primary goal was to examine the association of $\mathrm{HL}$ and time to discharge, which could not be evaluated among those who died. We excluded participants with LOS $>30$ days to limit overly influential effects of extreme outliers ( $1 \%$ of the population).

\section{Variables}

HL was screened using the Brief Health Literacy Screen (BHLS), a validated, 3-question verbal survey not requiring adequate visual acuity to assess HL. ${ }^{19,20}$ The 3 questions are as follows: (1) "How confident are you filling out medical forms by yourself?", (2) "How often do you have someone help you read hospital materials?", and (3) "How often do you have problems learning about your medical condition because of difficulty understanding written information?" Responses to the questions were scored on a 5-point Likert scale in which higher scores corresponded to higher HL. ${ }^{21,22}$ The scores for each of the 3 questions were summed to yield a range between 3 and 15. On the individual questions, prior work has demonstrated improved test performance with a cutoff of $\leq 3$, which corresponds to a response of "some of the time" or "somewhat"; therefore, when the 3 questions were summed together, scores of $\leq 9$ were considered indicative of low HL. ${ }^{21,23}$

For severity of illness adjustment, we used relative weights derived from the 3M (3M, Maplewood, MN) All Patient Refined Diagnosis Related Groups (APR-DRG) classification system, which uses administrative data to classify the severity. The APR-DRG system assigns each admission to a DRG based on principal diagnosis; for each DRG, patients are then subdivided into 4 severity classes based on age, comorbidity, and interactions between these variables and the admitting diagnosis. ${ }^{24}$ Using the base DRG and severity score, the system assigns relative weights that reflect differences in expected hospital resource utilization.

LOS was derived from hospital administrative data and counted from the date of admission to the hospital. Participants who were discharged on the day of admission were counted as having an LOS of 1 . Insurance status (Medicare, Medicaid, no payer, private) also was obtained from administrative data. Age, sex (male or female), education (junior high or less, some high school, high school graduate, some college, college graduate, postgraduate), and race (black/African American, white, Asian or Pacific Islander [including Asian Indian, Chinese, Filipino, Japanese, Korean, Vietnamese, other Asian, Native Hawaiian, Guam/Chamorro, Samoan, other Pacific], American Indian or Alaskan Native, multiple race) were obtained from administrative data based on information provided by the patient. Participants with missing data on any of the sociodemographic variables or on the APR-DRG score were excluded from the analysis.

\section{Statistical Analysis}

$\chi^{2}$ and 2-tailed $t$ tests were used to compare categorical and continuous variables, respectively. Multivariate linear regressions were employed to measure associations between the independent variables (HL, illness severity, race, gender, education, and insurance status) and the dependent variable, LOS. Independent variables were chosen for clinical significance and retained in the model regardless of statistical significance. The adjusted $R^{2}$ values of models with and without the HL variable included were reported to provide information on the contribution of HL to the overall model.

Because LOS was observed to be right skewed and residuals of the untransformed regression were observed to be non-normally distributed, the decision was made to natural $\log$ transform LOS, which is consistent with previous hospital LOS studies. ${ }^{16}$ Regression coefficients and confidence intervals were then transformed into percentage estimates using the following equation: $100\left(e^{\beta}-1\right)$. Adjusted $R^{2}$ was reported for the transformed regression.

The APR-DRG relative weight was treated as a continuous variable. Sociodemographic variables were dichotomized as follows: female vs male; high school graduates vs not; African American vs not; Medicaid/no payer vs Medicare/private payer. Age was not included in the multivariate model because it has been incorporated into the weighted APR-DRG illness severity scores.

Each of the sociodemographic variables and the APRDRG score were examined for effect modification via the same multivariate linear equation described above, with the addition of an interaction term. A separate regression was performed with an interaction term between age (dichotomized at 265 ) and HL to investigate whether age modified the association between HL and LOS. Finally, we explored whether effects were isolated to long vs short LOS by dividing the sample based on the mean LOS ( $\geq 6$ days) and performing separate multivariate comparisons.

Sensitivity analyses were performed to exclude those with LOS greater than the 90th percentile and those with APRDRG score greater than the 90th percentile; age was added to the model as a continuous variable to evaluate whether the illness severity score fully adjusted for the effects of age on LOS. Furthermore, we compared the participants with missing data to those with complete data across both dependent and independent variables. Alpha was set at 0.05; analyses were performed using Stata Version 14 (Stata, College Station, TX).

\section{RESULTS}

A total of 5983 participants met inclusion criteria and completed the HL assessment; of these participants, 75 (1\%) died during hospitalization, $9(0.2 \%)$ had missing discharge status, and $79(1 \%)$ had LOS $>30$ days. Two hundred eighty $(5 \%)$ were missing data on sociodemographic variables or APRDRG score. Of the remaining $(\mathrm{n}=5540)$, the mean age was 57 years (standard deviation $[\mathrm{SD}]=19$ years), over half of participants were female (57\%), and the majority were African American (73\%) and had graduated from high school (81\%). The sample was divided into those with private insurance (25\%), those with Medicare (46\%), and those with Medicaid (26\%); $2 \%$ had no payer. The mean APR-DRG score was 1.3 $(\mathrm{SD}=1.2)$, and the scores ranged from 0.3 to 15.8 .

On the BHLS screen for HL, 20\% (1104/5540) had inad- 
TABLE 1. Associations with Length of Stay

\begin{tabular}{|c|c|c|c|c|}
\hline \multirow[b]{2}{*}{ Characteristic } & \multicolumn{2}{|c|}{ Unadjusted } & \multicolumn{2}{|c|}{ Adjusted } \\
\hline & \% Change (95\% Cl) & $P$ Value & \% Change (95\% Cl) & $P$ Value \\
\hline \multicolumn{5}{|l|}{ HL } \\
\hline Adequate $\mathrm{HL}$ & Reference & & Reference & \\
\hline Low HL & 14.3 (8.5 to 20.1) & $<.001$ & $11.1(6.1$ to 16.1$)$ & $<.001$ \\
\hline \multicolumn{5}{|l|}{ Severity of Illness } \\
\hline APR-DRG, 1 point increase & 36.0 (34.1 to 38.0 ) & $<.001$ & 35.3 (33.3 to 37.2 ) & $<.001$ \\
\hline \multicolumn{5}{|l|}{ Sociodemographics } \\
\hline Female & $-5.9(-9.7$ to -2.0$)$ & .003 & 0.0 (-3.6 to 3.5$)$ & 1 \\
\hline Non-HS grad & $-4.1(-9.0$ to 0.9$)$ & .1 & $-3.3(-7.8$ to 1.2$)$ & .1 \\
\hline African American & $-16.1(-20.0$ to -12.3$)$ & $<.001$ & $-7.2(-11.0$ to -3.4$)$ & $<.001$ \\
\hline Medicaid/Uninsured & $-13.0(-16.9$ to -9.1$)$ & $<.001$ & $-7.4(-11.1$ to -3.7$)$ & $<.001$ \\
\hline
\end{tabular}

NOTE: Multivariate models adjusted for the following covariates: HL, APR-DRG score, gender, education, race, and insurance status. Abbreviations: Adj, adjusted; APR-DRG, all payer refined diagnosis related group; Cl, confidence interval; HL, health literacy; HS, high school.

equate HL. Participants with low HL had higher weighted illness severity scores (average 1.4 vs $1.3 ; P=0.003$ ). Participants with low HL were also more likely to be 65 or older (55\% vs 33\%; $P<0.001)$, non-high school graduates $(35 \%$ vs $15 \% ; P<0.001)$, and African American $(78 \%$ vs $72 \% ; P$ $<0.001$ ), and to have Medicare or private insurance ( $75 \%$ vs $71 \% ; P=0.02$ ). There was no significant difference with respect to gender ( $54 \%$ male vs $57 \%$ female; $P=0.1$ )

The mean and median LOS were $6 \pm 5$ days and 4 days (interquartile range 2-7 days), respectively. Those with low HL had a longer average LOS (6.0 vs 5.4 days; $P<0.001$ ). In multivariate analysis controlling for APR-DRG score, gender, education, race, and insurance status, low HL was associated with an $11.1 \%$ longer LOS (95\% CI, 6.1-16.1; P $<0.001$; Table 1). The adjusted $R^{2}$ value for the regression was $25.0 \%$ including $\mathrm{HL}$ and $24.7 \%$ with $\mathrm{HL}$ excluded. Additionally, being African American $(P<0.001)$ and having Medicaid or no insurance $(P<0.001)$ were associated with a shorter LOS in multivariate analysis (Table 1). The association of HL and LOS in multivariate modeling remained significant among participants with LOS $<6$ days $(10.2 \%$; $95 \%$ CI, 5.6\%-14.9\%; $P<0.001)$, but not among participants with LOS $\geq 6$ days $(0.4 \% ; 95 \% \mathrm{CI},-3.6 \%$ to $4.4 \% ; P=0.8)$.

Neither age $\geq 65(P=0.4)$ nor illness severity score $(P=$ $0.5)$ significantly modified the effect of $\mathrm{HL}$ on LOS. However, the effect of HL on hospital LOS was significantly modified by gender $(P=0.02)$. Among men, low HL was associated with a $17.8 \%$ longer LOS $(95 \% \mathrm{CI}, 10.0 \%-25.7 \%$; $P$ $<0.001$ ), but among women, low HL was associated with only a $7.7 \%$ longer LOS (95\% CI, $1.9 \%-13.5 \%$; $P=0.009$ ). Among the remaining demographics, high school graduation status $(P=0.4)$, being African American $(P=0.6)$, and insurance status $(P=0.2)$ did not significantly modify the effect of HL on LOS. In sensitivity analysis, excluding participants with LOS above the 90th percentile of 12 days and excluding participants with illness severity scores above the 90th percentile, low HL was still associated with longer LOS $(P<0.001$ and $P=0.001$, respectively; Table 2$)$. In the final sensitivity analysis, although age remained a significant predictor of longer LOS after controlling for illness severity (0.2\% increase per year, $95 \% \mathrm{CI}, 0.1 \%-0.3 \% ; P<0.001)$, low HL nevertheless remained significantly associated with longer LOS $(P<0.001$; Table 2$)$.

Finally, we compared the group with missing data $(\mathrm{n}=$ $280)$ to the group with complete data $(\mathrm{n}=5540)$. The participants with missing data were more likely to have low HL (31\% [86/280] vs $20 \% ; P<0.001)$ and to have Medicare or private insurance $(82 \%[177 / 217]$ vs $72 \% ; P=0.002)$; however, they were not more likely to be 65 or older $(40 \%$ [112/280] vs $37 \% ; P=0.3)$, high school graduates $(88 \%$ [113/129] vs $81 \% ; P=0.06)$, African American $(69 \%$ [177/256] vs $73 \% ; P=0.1)$, or female $(57 \%$ [158/279] vs $57 \% ; P=1)$, nor were they more likely to have longer LOS $(5.7[\mathrm{n}=280]$ vs 5.5 days; $P=0.6)$ or higher illness severity scores $(1.3[\mathrm{n}=231]$ vs $1.3 ; P=0.7)$.

\section{DISCUSSION}

To our knowledge, this study is the first to evaluate the association between low HL and an important in-hospital outcome measure, hospital LOS. We found that low HL was associated with a longer hospital LOS, a result which remained significant when controlling for severity of illness and sociodemographic variables and when testing the model for sensitivity to the highest values of LOS and illness severity. Additionally, the association of HL with LOS appeared concentrated among participants with shorter LOS. Relative to other predictors, the contribution of HL to the overall LOS model was small, as evidenced by the change in adjusted $R^{2}$ values with HL excluded.

Among the covariates, only gender modified the association between HL and LOS; the findings suggested that men were more susceptible to the effect of low HL on increased 
TABLE 2. Associations with Length of Stay: Sensitivity Analysis

\begin{tabular}{|c|c|c|c|c|c|c|}
\hline \multirow[b]{2}{*}{ Characteristic } & \multicolumn{2}{|c|}{ Excluding $>90$ th $\%$ LOS } & \multicolumn{2}{|c|}{ Exclude $>90$ th $\%$ APR-DRG } & \multicolumn{2}{|c|}{ Including Age } \\
\hline & $\%$ Change $(95 \% \mathrm{Cl})$ & $P$ Value & \% Change (95\% Cl) & $P$ Value & \% Change $(95 \% \mathrm{Cl})$ & $P$ Value \\
\hline \multicolumn{7}{|l|}{ HL } \\
\hline Adequate $\mathrm{HL}$ & Reference & & Reference & & Reference & \\
\hline Low HL & $8.8(4.1$ to 13.5$)$ & $<.001$ & 8.6 (3.6 to 13.7 ) & .001 & 9.1 (4.1 to 14.1$)$ & $<.001$ \\
\hline \multicolumn{7}{|l|}{ Severity of Illness } \\
\hline $\begin{array}{l}\text { APR-DRG, } 1 \\
\text { point increase }\end{array}$ & 33.1 (30.5 to 35.6) & $<.001$ & 87.7 (80.7 to 94.6) & $<.001$ & 35.1 (33.2 to 37.1$)$ & $<.001$ \\
\hline \multicolumn{7}{|l|}{ Sociodemographics } \\
\hline Female & $2.3(-1.2$ to 5.8$)$ & .2 & $1.7(-2.0$ to 5.3$)$ & .4 & $-0.4(-4.0$ to 3.1$)$ & .8 \\
\hline Non-HS grad & $-2.0(-6.3$ to 2.2$)$ & .4 & $-3.3(-7.8$ to 1.3$)$ & .2 & $-4.5(-8.9$ to -0.05$)$ & .048 \\
\hline African American & $-4.2(-8.0$ to -0.4$)$ & .03 & $-5.3(-9.4$ to -1.3$)$ & .009 & $-7.8(-11.6$ to -4.0$)$ & $<.001$ \\
\hline Medicaid/Uninsured & $-7.3(-10.8$ to -3.8$)$ & $<.001$ & $-6.7(-10.5$ to -2.9$)$ & .001 & $-3.7(-7.9$ to 0.6$)$ & .09 \\
\hline Age (1 year increase) & - & - & - & - & $0.2(0.1$ to 0.3$)$ & $<.001$ \\
\hline$N, \operatorname{Adj} R^{2}$ & $5079,15.5 \%$ & & $4988,19.6 \%$ & & $5540,25.2 \%$ & \\
\hline
\end{tabular}

LOS. Illness severity and other sociodemographics, including age $\geq 65$, did not appear to modify the association. We also found that being African American and having Medicaid or no insurance were associated with a significantly shorter LOS in multivariate analysis.

Previous work suggested that the adverse health effects of low HL may be mediated through several pathways, including health knowledge, self-efficacy, health skills, and illness stigma. ${ }^{25-27}$ The finding of a small but significant relationship between $\mathrm{HL}$ and LOS was not surprising given these known associations; nevertheless, there may be an additional patient-dependent effect of low HL on LOS not discovered here. For instance, patients with poor health knowledge and self-efficacy might stay in the hospital longer if they or their providers do not feel comfortable with their self-care ability.

This finding may be useful in developing hospital-based interventions. HL-specific interventions, several of which have been tested in the inpatient setting, ${ }^{14,28,29}$ have shown promise toward improving health knowledge, ${ }^{30}$ disease severity, ${ }^{31}$ and health resource utilization. ${ }^{32}$

Those with low HL may lack the self-efficacy to participate in discharge planning; in fact, previous work has related low HL to posthospital readmissions. ${ }^{8,9}$ Conversely, patients with low HL might struggle to engage in the inpatient milieu, advocating for shorter LOS if they feel alienated by the inpatient experience.

These possibilities show that LOS is a complex measure shown to depend on patient-level characteristics and on provider-based, geographical, and sociocultural factors. ${ }^{16,33}$ With these forces at play, additional effects of lower levels of HL may be lost without phenotyping patients by both level of $\mathrm{HL}$ and related characteristics, such as self-efficacy, health skills, and stigma. By gathering these additional data, future work should explore whether subpopulations of patients with low HL may be at risk for too-short vs too-long hospital admissions.

For instance, in this study, both race and Medicaid insurance were associated with shorter LOS. Being African American was associated with shorter LOS in our study but has been found to be associated with longer LOS in another study specifically focused on diabetes. ${ }^{34}$ Prior findings found uninsured patients have shorter LOS. ${ }^{35}$ Therefore, these findings in our study are difficult to explain without further work to understand whether there are health disparities in the way patients are cared for during hospitalization that may shorten or lengthen their LOS because of factors outside of their clinical need.

The finding that gender modified the effect of low HL on LOS was unexpected. There were similar proportions of men and women with low HL. There is evidence to support that women make the majority of health decisions for themselves and their familes ${ }^{36}$; therefore, there may be unmeasured aspects of HL that provide an advantage for female vs male inpatients. Furthermore, omitted confounders, such as social support, may not fully capture potential gender-related differences. Future work is needed to understand the role of gender in relationship to HL and LOS.

Limitations of this study include its observational, single-centered design with information derived from administrative data; positive and negative confounding cannot be ruled out. For instance, we did not control for complex aspects affecting LOS, such as discharge disposition and goals of care (eg, aggressive care after discharge vs hospice). To address this limitation, multivariate analyses were performed, which were adjusted for illness severity scores and took into account both comorbidity and severity of the current illness. Additionally, although it is important to study such populations, our largely urban, minority sample is not representative of the U.S. population, and within our large sample, 
there were participants with missing data who had lower $\mathrm{HL}$ on average, although this group represented only $5 \%$ of the sample. Finally, different HL tools have noncomplete concordance, which has been seen when comparing the BHLS with more objective tools. ${ }^{20,37}$ Furthermore, certain in-hospital clinical scenarios (eg, recent stroke or prolonged intensive care unit stay) may present unique challenges in establishing a baseline HL level. However, the BHLS was used in this study because of its greater feasibility.

In conclusion, this study is the first to evaluate the relationship between low HL and LOS. The findings suggest that HL may play a role in shaping outcomes in the inpatient setting and that targeting interventions toward screened patients may be a pathway toward mitigating adverse effects. Our findings need to be replicated in larger, more representative samples, and further work understanding subpopulations within the low HL population is needed. Future work should measure this association in diverse inpatient settings (eg, psychiatric, surgical, and specialty), in addition to assessing associations between $\mathrm{HL}$ and other important in-hospital outcome measures, including mortality and discharge disposition.

\section{Acknowledgments}

The authors thank the Hospitalist Project team for their assistance with data collection. The authors especially thank Chuanhong Liao and Ashley Snyder for assistance with statistical analyses; Andrea Flores, Ainoa Coltri, and Tom Best for their assistance with data management. The authors would also like to thank Nicole Twu for her help with preparing and editing the manuscript.

Disclosures: Dr. Jaffee was supported by a Calvin Fentress Research Fellowship and NIH R25MH094612. Dr. Press was supported by a career development award (NHLBI K23HL118151). This work was also supported by a seed grant from the Center for Health Administration Studies. All other authors declare no conflicts of interest.

\section{References}

1. U.S. Department of Health and Human Services. Healthy People 2010: Understanding and Improving Health. Washington, DC: U.S. Government Printing Office; 2000.

2. "What Did the Doctor Say"? Improving Health Literacy to Protect Patient Safety. The Joint Commission; 2007.

3. Kutner M, Greenberg E, Jin Y, Paulsen C. The Health Literacy of America's Adults: Results from the 2003 National Assessment of Adult Literacy. National Center for Education Statistics; 2006.

4. Davis TC, Wolf MS, Bass PF, et al. Literacy and misunderstanding prescription drug labels. Ann Intern Med. 2006;145(12):887-894.

5. Kripalani S, Henderson LE, Chiu EY, Robertson R, Kolm P, Jacobson TA. Predictors of medication self-management skill in a low-literacy population. J Gen Intern Med. 2006;21(8):852-856.

6. Berkman ND, Sheridan SL, Donahue KE, Halpern DJ, Crotty K. Low health literacy and health outcomes: an updated systematic review. Ann Intern Med. 2011;155(2):97-107.

7. Baker DW, Parker RM, Williams MV, Clark WS. Health literacy and the risk of hospital admission. J Gen Intern Med. 1998;13(12):791-798.

8. Mitchell SE, Sadikova E, Jack BW, Paasche-Orlow MK. Health literacy and 30-day postdischarge hospital utilization. J Health Commun. 2012;17(Suppl 3):325-338.

9. Jaffee EG, Arora VM, Matthiesen MI, Hariprasad SM, Meltzer DO, Press VG. Postdischarge Falls and Readmissions: Associations with Insufficient Vision and Low Health Literacy among Hospitalized Seniors. J Health Commun. 2016;21(sup2):135-140.

10. Hope CJ, Wu J, Tu W, Young J, Murray MD. Association of medication adherence, knowledge, and skills with emergency department visits by adults 50 years or older with congestive heart failure. Am J Health Syst Pharm. 2004;61(19): 2043-2049.

11. Bennett IM, Chen J, Soroui JS, White S. The contribution of health literacy to disparities in self-rated health status and preventive health behaviors in older adults. Ann Fam Med. 2009; 7(3):204-211.

12. Baker DW, Wolf MS, Feinglass J, Thompson JA. Health literacy, cognitive abilities, and mortality among elderly persons. J Gen Intern Med. 2008;23(6):723-726.

13. Cho YI, Lee SY, Arozullah AM, Crittenden KS. Effects of health literacy on health status and health service utilization amongst the elderly. Soc Sci Med. 2008;66(8):1809-1816.

14. Paasche-Orlow MK, Riekert KA, Bilderback A, et al. Tailored education may reduce health literacy disparities in asthma self-management. Am J Respir Crit Care Med. 2005;172(8):980-986.

15. Soria-Aledo V, Carrillo-Alcaraz A, Campillo-Soto Á, et al. Associated factors and cost of inappropriate hospital admissions and stays in a second-level hospital. Am J Med Qual. 2009;24(4):321-332.

16. Lu M, Sajobi T, Lucyk K, Lorenzetti D, Quan H. Systematic review of risk adjustment models of hospital length of stay (LOS). Med Care. 2015;53(4):355-365.

17. Clarke A, Rosen R. Length of stay. How short should hospital care be? Eur J Public Health. 2001;11(2):166-170.

18. Meltzer D, Manning WG, Morrison J, et al. Effects of physician experience on costs and outcomes on an academic general medicine service: results of a trial of hospitalists. Ann Intern Med. 2002;137(11):866-874.

19. Chew LD, Bradley KA, Boyko EJ. Brief questions to identify patients with inade-

quate health literacy. Fam Med. 2004;36(8):588-594.

20. Press VG, Shapiro MI, Mayo AM, Meltzer DO, Arora VM. More than meets the eye: relationship between low health literacy and poor vision in hospitalized patients. J Health Commun. 2013;18 Suppl 1:197-204.

21. Willens DE, Kripalani S, Schildcrout JS, et al. Association of brief health literacy screening and blood pressure in primary care. J Health Commun. 2013;18 Suppl 1:129-142.

22. Peterson PN, Shetterly SM, Clarke CL, et al. Health literacy and outcomes among patients with heart failure. JAMA. 2011;305(16):1695-1701.

23. Chew LD, Griffin JM, Partin MR, et al. Validation of screening questions for limited health literacy in a large VA outpatient population. J Gen Intern Med. 2008;23(5):561-566.

24. Averill RF, Goldfield N, Hughes JS, et al. All Patient Refined Diagnosis Related Groups (APR-DRGs): Methodology Overview. 3M Health Information Systems; 2003.

25. Waite KR, Paasche-Orlow M, Rintamaki LS, Davis TC, Wolf MS. Literacy, social stigma, and HIV medication adherence. J Gen Intern Med. 2008;23(9):1367-1372.

26. Paasche-Orlow MK, Wolf MS. The causal pathways linking health literacy to health outcomes. Am J Health Behav. 2007;31 Suppl 1:S19-26.

27. Berkman ND, Sheridan SL, Donahue KE, et al. Health literacy interventions and outcomes: an updated systematic review. Evid Rep Technol Assess (Full Rep). 2011;(199):1-941.

28. Kripalani S, Roumie CL, Dalal AK, et al. Effect of a pharmacist intervention on clinically important medication errors after hospital discharge: a randomized trial. Ann Intern Med. 2012;157(1):1-10.

29. Press VG, Arora VM, Shah LM, et al. Teaching the use of respiratory inhalers to hospitalized patients with asthma or COPD: a randomized trial. J Gen Intern Med. 2012;27(10):1317-1325.

30. Sobel RM, Paasche-Orlow MK, Waite KR, Rittner SS, Wilson EAH, Wolf MS. Asthma 1-2-3: a low literacy multimedia tool to educate African American adults about asthma. J Community Health. 2009;34(4):321-327.

31. Rothman RL, DeWalt DA, Malone R, et al. Influence of patient literacy on the effectiveness of a primary care-based diabetes disease management program. JAMA. 2004;292(14):1711-1716.

32. DeWalt DA, Malone RM, Bryant ME, et al. A heart failure self-management program for patients of all literacy levels: a randomized, controlled trial [ISRCTN11535170]. BMC Health Serv Res. 2006;6:30.

33. Hasan O, Orav EJ, Hicks LS. Insurance status and hospital care for myocardial infarction, stroke, and pneumonia. J Hosp Med. 2010;5(8):452-459.

34. Cook CB, Naylor DB, Hentz JG, et al. Disparities in diabetes-related hospitalizations: relationship of age, sex, and race/ethnicity with hospital discharges, lengths of stay, and direct inpatient charges. Ethn Dis. 2006;16(1):126-131.

35. Hadley J, Steinberg EP, Feder J. Comparison of uninsured and privately insured hospital patients. Condition on admission, resource use, and outcome. JAMA. 1991;265(3):374-379.

36. Women's Health Care Chartbook: Key Findings From the Kaiser Women's Health Survey. May 2011. https://kaiserfamilyfoundation.files.wordpress. com/2013/01/8164.pdf. Accessed August 1, 2017.

37. Louis AJ, Arora VM, Matthiesen MI, Meltzer DO, Press VG. Screening Hospitalized Patients for Low Health Literacy: Beyond the REALM of Possibility? Health Educ Behav. 2017;44(3):360-364. 